\title{
Immobilization of Rhodium Complexes at Thiolate Monolayers on Gold Surfaces: Catalytic and Structural Studies
}

\author{
Thomas Belser ${ }^{\dagger}$, Meike Stöhr ${ }^{+}, *$ and Andreas Pfaltz ${ }^{\dagger}, *$ \\ Contribution from the Department of Chemistry, ${ }^{\dagger}$ St. Johanns-Ring 19 and the Department of Physics, ${ }^{\dagger}$ \\ Klingelbergstrasse 82, University of Basel, 4056 Basel (Switzerland).
}

\section{SUPPORTING INFORMATION}

General Methods: Reactions with air- or moisture-sensitive compounds were performed under argon using standard Schlenk techniques or under purified $\mathrm{N}_{2}$ in a MBraun glovebox. Glasware was oven dried and flame dried prior to use. All chemicals were purchased from Fluka or Aldrich. $\mathrm{CH}_{2} \mathrm{Cl}_{2}$ was dried over $\mathrm{CaH}_{2}$ and $\mathrm{THF}$ over $\mathrm{Na} / \mathrm{K}$ and freshly distilled under a stream of nitrogen prior to use. Optical rotations were measured in a Perkin Elmer Polarimeter 341, sodium lamp, $1 \mathrm{dm}$ cuvette length, $c$ in g/100 mL. ${ }^{1} \mathrm{H}$-, ${ }^{13} \mathrm{C}$-, ${ }^{19} \mathrm{~F}$ - and ${ }^{31} \mathrm{P}-\mathrm{NMR}$ spectra were recorded in $\mathrm{CD}_{2} \mathrm{Cl}_{2}$ or $\mathrm{CDCl}_{3}$ on a Bruker Avance $400 \mathrm{MHz}$ or $500 \mathrm{MHz}$ spectrometer and coupling constants are reported in Hz. Chemical shifts are given in ppm relative to TMS. IR spectra: 1600 Perkin Elmer Series FT-IR spectrometer; FAB mass spectra: Finnigan MAT 312; EI mass spectra: VG 70 SE. Elemental analysis were carried out on a Leco CHN-900 and Leco RO-478. Chromatographic purifications were performed by flash chromatography using silica gel (Merck $0.040-0.063 \mathrm{~mm}$ ). Yields for final products refer to isolated products and are the average of three runs. GC analyses: Carlo Erba HRGC Mega2 Series MFC 800 (column: Restek Rtx-1701; $0.25 \mathrm{~mm}, 30 \mathrm{~m}, 60 \mathrm{kPa} H \mathrm{He}$ ). HPLC analyses: Shimadzu VP-system (column: Daicel OD-H; $4.6 \times 250 \mathrm{~mm})$.

\section{Synthesis of 11-(10'-carboxy-decyldisulfanyl)undecanoic acid}

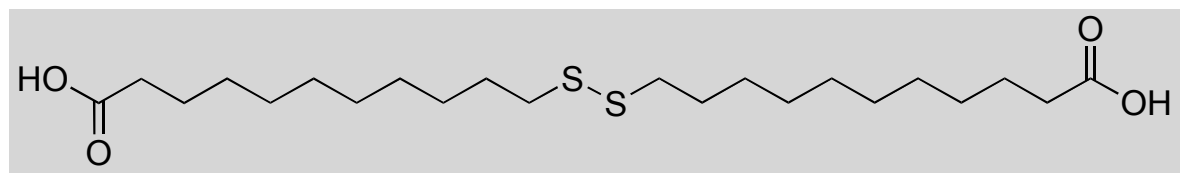

Sodium hydroxide $(366 \mathrm{mg}, 9.16 \mathrm{mmol})$, potassium iodide $(50.0 \mathrm{mg}, 0.30 \mathrm{mmol})$ and iodine $(1.16 \mathrm{~g}$, $4.58 \mathrm{mmol})$ were added to a solution of 11-mercaptoundecanoic acid (2.00 g, $9.16 \mathrm{mmol})$ in methanol $(50 \mathrm{~mL})$ and the solution was stirred for $30 \mathrm{~min}$. The brown reaction mixture was decolored with a saturated sodium sulfite solution, the solvents concentrated under reduced pressure, the precipitate dissolved in $\mathrm{CH}_{2} \mathrm{Cl}_{2}(50 \mathrm{~mL})$ and the resulting solution washed with a $\mathrm{HCl}$ solution $(1 \mathrm{M}, 50 \mathrm{~mL})$ and water $(50 \mathrm{~mL})$. The organic phase was dried over $\mathrm{MgSO}_{4}$, filtered and concentrated under reduced 
pressure. Purification of the crude material by crystallization (hexanes/AcOEt) gave 63\% yield of the desired 11-(10'-carboxy-decyldisulfanyl)undecanoic acid (1.25 g).

m.p.: $68-70{ }^{\circ} \mathrm{C} . \mathrm{R}_{\mathrm{f}}=0.30$ (hexanes/AcOEt $=4: 1$ ).

${ }^{1} \mathrm{H}-\mathrm{NMR}\left(400.1 \mathrm{MHz}, \mathrm{CDCl}_{3}, 295 \mathrm{~K}\right): \delta=1.28\left(\mathrm{~s}_{\mathrm{b}}, 20 \mathrm{H}, \mathrm{CH}_{2}\right), 1.35\left(\mathrm{~m}_{\mathrm{c}}, 4 \mathrm{H}, \mathrm{CH}_{2}\right), 1.64\left(\mathrm{~m}_{\mathrm{c}}\right.$, $\left.J=7.4 \mathrm{~Hz}, 8 \mathrm{H}, \mathrm{CH}_{2} \mathrm{CH}_{2} \mathrm{CO}_{2} \mathrm{H}+\mathrm{SCH}_{2} \mathrm{CH}_{2}\right), 2.34\left(\mathrm{t}, J=7.4 \mathrm{~Hz}, 4 \mathrm{H}, \mathrm{CH}_{2} \mathrm{CO}_{2} \mathrm{H}\right), 2.68(\mathrm{t}, J=7.4 \mathrm{~Hz}$, $\left.4 \mathrm{H}, \mathrm{SCH}_{2}\right), 10.98\left(\mathrm{~s}_{\mathrm{b}}, 2 \mathrm{H}, \mathrm{CO}_{2} \mathrm{H}\right)$.

${ }^{13} \mathrm{C}\left\{{ }^{1} \mathrm{H}\right\}-\mathrm{NMR}\left(100.6 \mathrm{MHz}, \mathrm{CDCl}_{3}, 295 \mathrm{~K}\right): \delta=25.0\left(\mathrm{CH}_{2}\right), 28.9\left(\mathrm{CH}_{2}\right), 29.4\left(\mathrm{CH}_{2}\right), 29.5\left(C \mathrm{H}_{2}\right), 29.5$ $\left(\mathrm{CH}_{2}\right), 29.6\left(\mathrm{CH}_{2}\right), 29.7\left(\mathrm{CH}_{2}\right), 29.8\left(\mathrm{CH}_{2}\right), 34.4\left(\mathrm{CH}_{2}\right), 39.6\left(\mathrm{SCH}_{2}\right), 180.7\left(\mathrm{CO}_{2} \mathrm{H}\right)$.

IR (KBr): $v\left(\mathrm{~cm}^{-1}\right)=2918 \mathrm{~s}, 2849 \mathrm{~s}, 1696 \mathrm{~s}$, $1471 \mathrm{~m}, 1427 \mathrm{~m}, 1289 \mathrm{~m}, 1261 \mathrm{~m}, 1231 \mathrm{~m}, 1206 \mathrm{~m}, 944 \mathrm{~m}$.

MS (FAB): $m / z\left(\right.$ rel int \%) = $434\left(\mathrm{M}^{+}, 30\right), 417$ (20), 344 (34), 328 (15), 232 (13), 171 (15), 127 (14), 111 (27), 101 (25), 87 (57), 81 (32), 69 (49), 55 (100), 43 (47).

Elemental analysis for $\mathrm{C}_{22} \mathrm{H}_{42} \mathrm{O}_{4} \mathrm{~S}_{2}$ calc.: C 60.79\%, $\mathrm{H} 9.74 \%$, O 14.72\%; found: $\mathrm{C} 60.84 \%, \mathrm{H} 9.68 \%$, O 14.74\%.

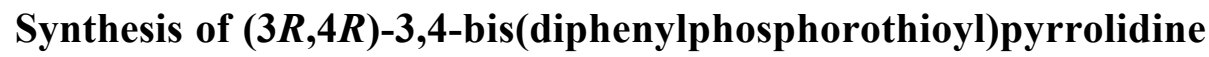

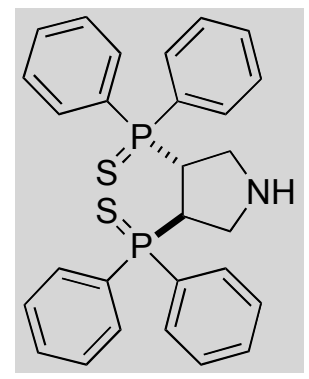

Sulfur $(0.27 \mathrm{~g}, 8.40 \mathrm{mmol})$ was added to a solution of PYRPHOS $(1.85 \mathrm{~g}, 4.20 \mathrm{mmol})$ in freshly distilled toluene $(50 \mathrm{~mL})$ and the reaction mixture was stirred for 12 hours at $23{ }^{\circ} \mathrm{C}$. The resulting solution was concentrated under reduced pressure. Purification of the crude material by flash chromatography, eluting with diethyl ether, gave $95 \%$ yield of the desired compound $(2.01 \mathrm{~g})$.

m.p.: $212-214{ }^{\circ} \mathrm{C} .[\alpha]_{\mathrm{p}}^{20}=+12.3\left(\mathrm{c}=0.80, \mathrm{CHCl}_{3}\right) . \mathrm{R}_{\mathrm{f}}=0.50$ (diethyl ether).

${ }^{1} \mathrm{H}-\mathrm{NMR}\left(400.1 \mathrm{MHz}, \mathrm{CDCl}_{3}, 295 \mathrm{~K}\right): \delta=2.59\left(\mathrm{~s}_{\mathrm{b}}, 1 \mathrm{H}, \mathrm{NH}\right), 3.12\left(\mathrm{~m}_{\mathrm{c}}, 2 \mathrm{H}, \mathrm{NCH}_{2}\right), 3.27\left(\mathrm{~m}_{\mathrm{c}}, 2 \mathrm{H}\right.$, $\left.\mathrm{NCH}_{2}\right), 4.01\left(\mathrm{~m}_{\mathrm{c}}, 2 \mathrm{H}, \mathrm{PCH}\right), 7.00\left(\mathrm{~m}_{\mathrm{c}}, 4 \mathrm{H}, \mathrm{CH} H_{\mathrm{Ph}}\right), 7.17\left(\mathrm{~m}_{\mathrm{c}}, 2 \mathrm{H}, \mathrm{CH}_{\mathrm{Ph}}\right), 7.42\left(\mathrm{~m}_{\mathrm{c}}, 6 \mathrm{H}, \mathrm{CH}_{\mathrm{Ph}}\right), 7.60$ $\left(\mathrm{m}_{\mathrm{c}}, 4 \mathrm{H}, \mathrm{C} H_{\mathrm{Ph}}\right), 7.88\left(\mathrm{~m}_{\mathrm{c}}, 4 \mathrm{H}, \mathrm{CH}_{\mathrm{Ph}}\right)$.

${ }^{13} \mathrm{C}\left\{{ }^{1} \mathrm{H}\right\}-\mathrm{NMR}\left(100.6 \mathrm{MHz}, \mathrm{CDCl}_{3}, 295 \mathrm{~K}\right): \delta=39.3-40.1(\mathrm{dd}, \mathrm{PCH}), 53.1\left(\mathrm{NCH}_{2}\right), 128.3-128.8$ $\left(\mathrm{m}, \mathrm{CH}_{\mathrm{Ph}-\text { meta }}\right), 130.0-132.9$ (m, $\left.C_{\mathrm{Ph}-\text { ipso }}\right), 130.9$ - $131.6\left(\mathrm{~m}, \mathrm{CH}_{\mathrm{Ph} \text {-ortho and para }}\right)$.

${ }^{31} \mathrm{P}\left\{{ }^{1} \mathrm{H}\right\}-\mathrm{NMR}\left(162.0 \mathrm{MHz}, \mathrm{CDCl}_{3}, 295 \mathrm{~K}\right): \delta=51.2$ (s).

IR $(\mathrm{KBr}): v\left(\mathrm{~cm}^{-1}\right)=3300 \mathrm{~m}, 3048 \mathrm{~m}, 2898 \mathrm{~m}, 1479 \mathrm{~m}, 1435 \mathrm{~s}, 1311 \mathrm{~m}, 1233 \mathrm{w}, 1158 \mathrm{~m}, 1099 \mathrm{~s}, 998 \mathrm{~m}, 861 \mathrm{~s}$, $745 \mathrm{~s}, 718 \mathrm{~s}, 693 \mathrm{~s}, 646 \mathrm{~s}, 605 \mathrm{~m}, 566 \mathrm{~m}, 521 \mathrm{~s}, 503 \mathrm{~s}, 489 \mathrm{~s}$.

MS (FAB): $m / z\left(\right.$ rel int \%) = $504\left([\mathrm{M}+\mathrm{H}]^{+}, 30\right), 286(9), 217(15), 185$ (6), 77 (5), 68 (100), 39 (5).

Elemental analysis for $\mathrm{C}_{28} \mathrm{H}_{27} \mathrm{NP}_{2} \mathrm{~S}_{2}$ calc.: C 66.78\%, H 5.40\%, N 2.78\%; found: $\mathrm{C} \mathrm{66.72 \% ,} \mathrm{H} \mathrm{5.32 \% ,}$ N $2.75 \%$. 


\section{SYNTHESIS OF THE SPACERS FOR THE GOLD COLLOIDS}

\section{Synthesis of 1-(2'-bromo-ethyl)adamantane}

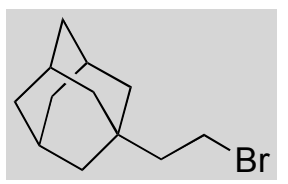

$N$-Bromosuccinimide (987 mg, $5.55 \mathrm{mmol}$ ) and triphenylphosphine (1.46 g, $5.55 \mathrm{mmol})$ were added to a solution of 2-adamant-1-yl-ethanol (1.00 g, $5.55 \mathrm{mmol})$ in freshly distilled benzene $(20 \mathrm{~mL})$ and the reaction mixture was stirred for 12 hours at $23{ }^{\circ} \mathrm{C}$. The resulting solution was washed with a saturated $\mathrm{NaCl}$ solution $(20 \mathrm{~mL})$ and water $(20 \mathrm{~mL})$. The organic phase was dried over $\mathrm{MgSO}_{4}$, filtered and concentrated under reduced pressure. Purification of the crude material by flash chromatography, eluting with $n$-pentane, gave $92 \%$ yield of 1-(2'-bromo-ethyl)adamantane $(1.24 \mathrm{~g})$.

m.p.: $66-68^{\circ} \mathrm{C} . \mathrm{R}_{\mathrm{f}}=0.78$ (n-pentane).

${ }^{1} \mathrm{H}-\mathrm{NMR}\left(400.1 \mathrm{MHz}, \mathrm{CDCl}_{3}, 295 \mathrm{~K}\right): \delta=1.51\left(\mathrm{~m}_{\mathrm{c}}, J=2.5 \mathrm{~Hz}, 6 \mathrm{H}, \mathrm{CCH}_{2-\mathrm{Ad}}\right), 1.61-1.70(\mathrm{~m}, 6 \mathrm{H}$, $\left.\mathrm{CH}_{2-\mathrm{Ad}}\right), 1.72\left(\mathrm{~m}_{\mathrm{c}}, 2 \mathrm{H}, \mathrm{BrCH}_{2} \mathrm{CH}_{2}\right), 1.96\left(\mathrm{~m}_{\mathrm{c}}, 3 \mathrm{H}, \mathrm{CH}_{\mathrm{Ad}}\right), 3.40\left(\mathrm{~m}_{\mathrm{c}}, 2 \mathrm{H}, \mathrm{BrCH}_{2}\right)$.

${ }^{13} \mathrm{C}\left\{{ }^{1} \mathrm{H}\right\}$-NMR $\left(100.6 \mathrm{MHz}, \mathrm{CDCl}_{3}, 295 \mathrm{~K}\right): \delta=28.6\left(\mathrm{CH}_{\mathrm{Ad}}\right), 29.2\left(\mathrm{BrCH}_{2}\right), 34.1\left(C_{\mathrm{Ad}}\right), 37.1\left(C \mathrm{H}_{2-\mathrm{Ad}}\right)$, $42.2\left(\mathrm{CCH}_{2-\mathrm{Ad}}\right), 48.2\left(\mathrm{BrCH}_{2} \mathrm{CH}_{2}\right)$.

IR $(\mathrm{KBr}): v\left(\mathrm{~cm}^{-1}\right)=2896 \mathrm{~s}, 2843 \mathrm{~s}, 2652 \mathrm{w}, 1452 \mathrm{~s}, 1442 \mathrm{~m}, 1344 \mathrm{~m}, 1329 \mathrm{~m}, 1312 \mathrm{~m}, 1256 \mathrm{~m}, 1215 \mathrm{~m}$, $1094 \mathrm{~m}, 973 \mathrm{~m}, 810 \mathrm{~m}, 746 \mathrm{w}, 719 \mathrm{~m}, 658 \mathrm{~m}, 560 \mathrm{~s}$.

MS (EI): $m / z$ (rel int \%) = $242\left(\mathrm{M}^{+}, 1,{ }^{79} \mathrm{Br}\right), 135$ (100), 107 (6), 93 (13), 79 (15), 67 (6), 55 (3), 41 (6).

Elemental analysis for $\mathrm{C}_{12} \mathrm{H}_{19} \mathrm{Br}$ calc.: C 59.27\%, H 7.88\%; found: C 59.22\%, H 7.79\%.

\section{Synthesis of 1-(hex-5'-enyl)adamantane}

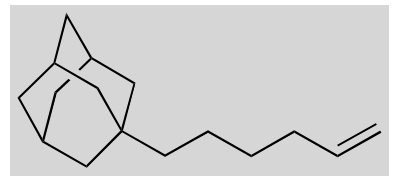

3-Butenylmagnesium bromide solution $(0.5 \mathrm{M}$ in THF, $94 \mathrm{~mL}, 47.0 \mathrm{mmol})$ was added tropwise at $-78{ }^{\circ} \mathrm{C}$ to a solution of 1-(2'-bromo-ethyl)adamantane $(5.00 \mathrm{~g}, 20.6 \mathrm{mmol})$ in freshly distilled THF $(30 \mathrm{~mL})$. After $10 \mathrm{~min}$, a lithium tetrachlorocuprate(II) solution $(0.1 \mathrm{M}$ in $\mathrm{THF}, 2.10 \mathrm{~mL}, 0.21 \mathrm{mmol})$ was added and the reaction mixture was stirred for 2 hours at $-78{ }^{\circ} \mathrm{C}$ and slowly warmed up to $23{ }^{\circ} \mathrm{C}$ over 12 hours. The resulting solution was washed with a saturated $\mathrm{NH}_{4} \mathrm{Cl}$ solution $(100 \mathrm{~mL})$ and water $(100 \mathrm{~mL})$. The organic phase was dried over $\mathrm{MgSO}_{4}$, filtered and concentrated under reduced pressure. Purification of the crude material by flash chromatography, eluting with $n$-pentane, gave $92 \%$ yield of 1-(hex-5'-enyl)adamantane (4.13 g).

$\mathrm{R}_{\mathrm{f}}=0.86$ (hexanes).

${ }^{1} \mathrm{H}-\mathrm{NMR}\left(400.1 \mathrm{MHz}, \mathrm{CDCl}_{3}, 295 \mathrm{~K}\right): \delta=1.03\left(\mathrm{t}, J=7.1 \mathrm{~Hz}, 2 \mathrm{H}, \mathrm{CCH}_{2}\right.$ ), 1.24 (quin, $J=7.1 \mathrm{~Hz}$, $2 \mathrm{H}, \mathrm{CCH}_{2} \mathrm{CH}_{2}$ ), 1.33 (quin, $J=7.1 \mathrm{~Hz}, 2 \mathrm{H}, \mathrm{CCH}_{2} \mathrm{CH}_{2} \mathrm{CH}_{2}$ ), $1.45\left(\mathrm{~m}_{\mathrm{c}}, J=2.2 \mathrm{~Hz}, 6 \mathrm{H}, \mathrm{CCH}_{2-\mathrm{Ad}}\right.$ ), 1.66 
$\left(\mathrm{m}_{\mathrm{c}}, 6 \mathrm{H}, \mathrm{CH} \mathrm{H}_{2-\mathrm{Ad}}\right), 1.93\left(\mathrm{~m}_{\mathrm{c}}, 3 \mathrm{H}, \mathrm{CH} \mathrm{Ad}\right), 2.05\left(\mathrm{~m}_{\mathrm{c}}, 2 \mathrm{H}, \mathrm{CCH}_{2} \mathrm{CH}_{2} \mathrm{CH}_{2} \mathrm{CH}_{2}\right) 4.96\left(\mathrm{~m}_{\mathrm{c}}, 2 \mathrm{H}, \mathrm{CH}_{2-\text { vinyl }}\right)$, $5.81\left(\mathrm{~m}_{\mathrm{c}}, 1 \mathrm{H}, \mathrm{C} H_{\text {vinyl }}\right)$.

${ }^{13} \mathrm{C}\left\{{ }^{1} \mathrm{H}\right\}-\mathrm{NMR}\left(100.6 \mathrm{MHz}, \mathrm{CDCl}_{3}, 295 \mathrm{~K}\right): \delta=22.0\left(\mathrm{CH}_{2}\right), 28.9\left(\mathrm{CH}_{\mathrm{Ad}}\right), 30.0\left(\mathrm{CH}_{2}\right), 32.4\left(C_{\mathrm{Ad}}\right), 34.0$ $\left(\mathrm{CH}_{2}\right), 37.4\left(\mathrm{CH}_{2-\mathrm{Ad}}\right), 42.7\left(\mathrm{CCH}_{2-\mathrm{Ad}}\right), 44.7\left(\mathrm{CH}_{2}\right), 114.2\left(\mathrm{CH}_{2 \text {-vinyl }}\right), 139.4\left(\mathrm{CH}_{\text {vinyl }}\right)$.

IR (NaCl): $v\left(\mathrm{~cm}^{-1}\right)=3076 \mathrm{w}, 2898 \mathrm{~s}, 2845 \mathrm{~s}, 2656 \mathrm{w}, 1820 \mathrm{w}, 1640 \mathrm{~m}, 1450 \mathrm{~m}, 1098 \mathrm{w}, 991 \mathrm{~m}, 908 \mathrm{~m}$.

MS (EI): $m / z$ (rel int \%) = $218\left(\mathrm{M}^{+}, 3\right), 135$ (100), 107 (4), 93 (8), 79 (9), 67 (5), 55 (4), 41 (5).

Elemental analysis for $\mathrm{C}_{16} \mathrm{H}_{26}$ calc.: C $88.00 \%$, H $12.00 \%$; found: C $88.07 \%, \mathrm{H} 12.01 \%$.

\section{Synthesis of thioacetic acid (S)-6-(adamant-1'-yl)hexyl ester}

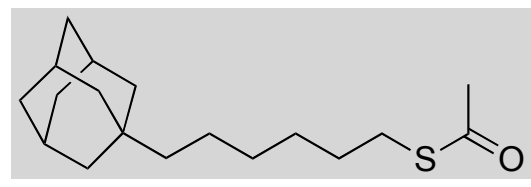

Thioacetic acid $(4.21 \mathrm{~mL}, 58.9 \mathrm{mmol})$ was added to a solution of 1-(hex-5'-enyl)adamantane (5.00 g, $22.9 \mathrm{mmol})$ in freshly distilled THF $(250 \mathrm{~mL})$ and cooled to $20^{\circ} \mathrm{C}$ with a cryostate (cooling bath: $\mathrm{H}_{2} \mathrm{O} / \mathrm{EtOH}=80: 20$ ). The solution was irradiated during 7 hours with a $300 \mathrm{~W}$ lamp (Osram UltraVitalux $300 \mathrm{~W}$ ). During that time was added 2,2'-azobisisobutyronitrile (900 $\mathrm{mg}, 5.48 \mathrm{mmol}$ ) in freshly distilled THF $(20 \mathrm{~mL})$ with a syringe pump. Irradiation was continued for another 4 hours. The solvent was concentrated under reduced pressure and the residue dissolved in dichloromethane $(100 \mathrm{~mL})$. The resulting solution was washed with a saturated $\mathrm{Na}_{2} \mathrm{CO}_{3}$ solution $(100 \mathrm{~mL})$ and water $(100 \mathrm{~mL})$. The organic phase was dried over $\mathrm{MgSO}_{4}$, filtered and concentrated under reduced pressure. Purification of the crude material by flash chromatography, eluting with $5 \%$ diethyl ether in $n$-pentane, gave $82 \%$ yield of thioacetic acid (S)-6-(adamant-1'-yl)hexyl ester (5.50 g).

$\mathrm{R}_{\mathrm{f}}=0.67$ (n-pentane/diethyl ether $\left.=20: 1\right)$.

${ }^{1} \mathrm{H}-\mathrm{NMR}\left(400.1 \mathrm{MHz}, \mathrm{CDCl}_{3}, 295 \mathrm{~K}\right): \delta=1.00\left(\mathrm{t}, J=7.3 \mathrm{~Hz}, 2 \mathrm{H}, \mathrm{CCH}_{2}\right), 1.22\left(\mathrm{~m}_{\mathrm{c}}, 4 \mathrm{H}, \mathrm{CH}_{2}\right), 1.35$ (quin, $\left.J=7.3 \mathrm{~Hz}, 2 \mathrm{H}, \mathrm{CCH}_{2} \mathrm{CH}_{2}\right), 1.43\left(\mathrm{~m}_{\mathrm{c}}, J=2.3 \mathrm{~Hz}, 6 \mathrm{H}, \mathrm{CCH}_{2-\mathrm{Ad}}\right.$ ), 1.55 (quin, $J=7.3 \mathrm{~Hz}, 2 \mathrm{H}$, $\left.\mathrm{CH}_{2} \mathrm{CH}_{2} \mathrm{~S}\right), 1.64\left(\mathrm{~m}_{\mathrm{c}}, 6 \mathrm{H}, \mathrm{CH}_{2-\mathrm{Ad}}\right), 1.92\left(\mathrm{~m}_{\mathrm{c}}, 3 \mathrm{H}, \mathrm{CH}_{\mathrm{Ad}}\right), 2.31\left(\mathrm{~s}, 3 \mathrm{H}, \mathrm{SCOCH}_{3}\right), 2.85(\mathrm{t}, J=7.3 \mathrm{~Hz}$, $\left.2 \mathrm{H}, \mathrm{CH}_{2} \mathrm{~S}\right)$.

${ }^{13} \mathrm{C}\left\{{ }^{1} \mathrm{H}\right\}$-NMR $\left(100.6 \mathrm{MHz}, \mathrm{CDCl}_{3}, 295 \mathrm{~K}\right): \delta=22.3\left(\mathrm{CH}_{2}\right), 28.8\left(\mathrm{CH}_{\mathrm{Ad}}\right), 29.0\left(\mathrm{CH}_{2}\right), 29.3\left(C \mathrm{H}_{2}\right)$, $29.6\left(\mathrm{CH}_{2}\right), 30.2\left(\mathrm{CH}_{2}\right), 30.7\left(\mathrm{CH}_{3}\right), 32.3\left(\mathrm{C}_{\mathrm{Ad}}\right), 37.4\left(\mathrm{CH}_{2-\mathrm{Ad}}\right), 42.6\left(\mathrm{CCH}_{2-\mathrm{Ad}}\right), 44.7\left(\mathrm{CH}_{2}\right), 196.1$ ( $\mathrm{SCO})$.

IR $(\mathrm{NaCl}): v\left(\mathrm{~cm}^{-1}\right)=2902 \mathrm{~s}, 2847 \mathrm{~s}, 2661 \mathrm{w}, 1694 \mathrm{~s}, 1450 \mathrm{~m}, 1352 \mathrm{~m}, 1132 \mathrm{~m}, 954 \mathrm{~m}, 864 \mathrm{w}, 624 \mathrm{w}$. MS (EI): $m / z\left(\right.$ rel int \%) = $294\left(\mathrm{M}^{+}, 4\right), 251$ (5), 135 (100), 107 (5), 93 (9), 79 (10), 43 (15).

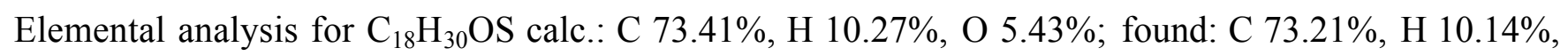
O $5.57 \%$. 


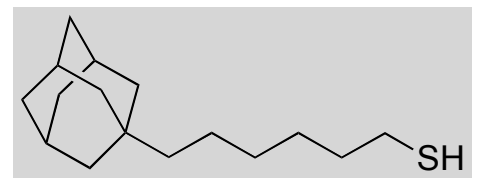

Conc. $\mathrm{HCl}(37 \%, 425 \mathrm{~mL}, 5.18 \mathrm{mmol})$ was added to a solution of thioacetic acid (S)-6-(adamant-1'-yl)hexyl ester $(150 \mathrm{mg}, 509 \mathrm{mmol})$ in methanol $(5 \mathrm{~mL})$ and the reaction mixture was stirred for 10 hours at $65{ }^{\circ} \mathrm{C}$. The solvent was concentrated under reduced pressure and the residue dissolved in diethyl ether $(20 \mathrm{~mL})$. The resulting solution was washed with a $\mathrm{HCl}$ solution $(1 \mathrm{M}, 20 \mathrm{~mL})$ and water $(20 \mathrm{~mL})$. The organic phase was dried over $\mathrm{MgSO}_{4}$, filtered and concentrated under reduced pressure. Purification of the crude material by flash chromatography, eluting with $n$-pentane, gave $86 \%$ yield of 6 -(adamant-1'yl)-hexanethiol $(0.11 \mathrm{~g})$.

$\mathrm{R}_{\mathrm{f}}=0.65$ (n-pentane).

${ }^{1} \mathrm{H}-\mathrm{NMR}\left(400.1 \mathrm{MHz}, \mathrm{CDCl}_{3}, 295 \mathrm{~K}\right): \delta=1.01\left(\mathrm{t}, J=7.4 \mathrm{~Hz}, 2 \mathrm{H}, \mathrm{CCH}_{2}\right), 1.22\left(\mathrm{~m}_{\mathrm{c}}, 4 \mathrm{H}, \mathrm{CH}_{2}\right), 1.32$ (t, $J=7.7 \mathrm{~Hz}, 1 \mathrm{H}, \mathrm{SH}$ ), 1.38 (quin, $J=7.4 \mathrm{~Hz}, 2 \mathrm{H}, \mathrm{CCH}_{2} \mathrm{CH}_{2}$ ), $1.44\left(\mathrm{~m}_{\mathrm{c}}, J=2.5 \mathrm{~Hz}, 6 \mathrm{H}, \mathrm{CCH}_{2-\mathrm{Ad}}\right.$ ), 1.60 (quin, $\left.J=7.4 \mathrm{~Hz}, 2 \mathrm{H}, \mathrm{CH}_{2} \mathrm{CH}_{2} \mathrm{SH}\right), 1.64\left(\mathrm{~m}_{\mathrm{c}}, 6 \mathrm{H}, \mathrm{CH}_{2-\mathrm{Ad}}\right), 1.92\left(\mathrm{~m}_{\mathrm{c}}, 3 \mathrm{H}, \mathrm{CH}_{\mathrm{Ad}}\right), 2.51$ (q, $\left.J=7.4 \mathrm{~Hz}, 2 \mathrm{H}, \mathrm{CH}_{2} \mathrm{SH}\right)$.

${ }^{13} \mathrm{C}\left\{{ }^{1} \mathrm{H}\right\}$-NMR $\left(100.6 \mathrm{MHz}, \mathrm{CDCl}_{3}, 295 \mathrm{~K}\right): \delta=22.3\left(\mathrm{CH}_{2}\right), 24.8\left(\mathrm{CH}_{2}\right), 28.5\left(\mathrm{CH}_{2}\right), 28.9\left(\mathrm{CH}_{\mathrm{Ad}}\right)$, $30.1\left(\mathrm{CH}_{2}\right), 32.3\left(\mathrm{C}_{\mathrm{Ad}}\right), 34.2\left(\mathrm{CH}_{2}\right), 37.4\left(\mathrm{CH}_{2}\right), 42.6\left(\mathrm{CH}_{\mathrm{Ad}}\right), 44.8\left(\mathrm{CH}_{2}\right)$.

IR $(\mathrm{NaCl}): v\left(\mathrm{~cm}^{-1}\right)=2902 \mathrm{~s}, 2846 \mathrm{~s}, 2663 \mathrm{w}, 1450 \mathrm{~m}, 1347 \mathrm{w}, 1101 \mathrm{w}$.

MS (EI): $m / z$ (rel int \%) = $252\left(\mathrm{M}^{+}, 3\right), 135$ (100), 93 (11), 79 (11), 67 (6), 41 (6).

Elemental analysis for $\mathrm{C}_{16} \mathrm{H}_{28} \mathrm{~S}$ calc.: $\mathrm{C} 76.12 \%, \mathrm{H} 11.18 \%$; found: $\mathrm{C} 76.07 \%, \mathrm{H} 11.08 \%$.

Synthesis of 11-aminoundecanol

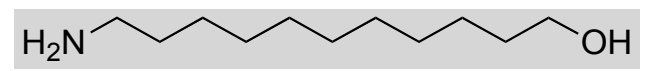

11-Aminoundecanoic acid $(21.0 \mathrm{~g}, 102 \mathrm{mmol})$ was added slowly at $-20{ }^{\circ} \mathrm{C}$ to a solution of $\mathrm{LiAlH}_{4}$ $(5.20 \mathrm{~g}, 137 \mathrm{mmol})$ in freshly distilled THF $(200 \mathrm{~mL})$ and the reaction mixture was stirred for 6 hours at $67{ }^{\circ} \mathrm{C}$. The solution was cooled to $0{ }^{\circ} \mathrm{C}$, treated with a $\mathrm{NaOH}$ solution $(10 \%, 10 \mathrm{~mL})$ and $\mathrm{H}_{2} \mathrm{O}(20 \mathrm{~mL})$ and stirred for $30 \mathrm{~min}$ at $23{ }^{\circ} \mathrm{C}$. The reaction mixture was dried over $\mathrm{MgSO}_{4}$, filtered and concentrated under reduced pressure to provide the desired 11-aminoundecanol $(10.3 \mathrm{~g})$ in 53\% yield.

m.p.: $66-68^{\circ} \mathrm{C}$.

${ }^{1} \mathrm{H}-\mathrm{NMR}\left(400.1 \mathrm{MHz}, \mathrm{CD}_{2} \mathrm{Cl}_{2}, 295 \mathrm{~K}\right): \delta=1.27\left(\mathrm{~s}_{\mathrm{b}}, 14 \mathrm{H}, \mathrm{CH}\right.$ ), 1.39 (quin, $J=7.0 \mathrm{~Hz}, 2 \mathrm{H}$, $\mathrm{CH}_{2} \mathrm{CH}_{2} \mathrm{NH}_{2}$ ), 1.50 (quin, $J=6.5 \mathrm{~Hz}, 2 \mathrm{H}, \mathrm{CH}_{2} \mathrm{CH}_{2} \mathrm{OH}$ ), 2.61 (t, $J=7.0 \mathrm{~Hz}, 2 \mathrm{H}, \mathrm{CH}_{2} \mathrm{NH}_{2}$ ), 3.54 (t, $\left.J=6.5 \mathrm{~Hz}, 2 \mathrm{H}, \mathrm{CH}_{2} \mathrm{OH}\right) \Rightarrow \mathrm{OH}$ und $\mathrm{NH}_{2}$ signals lies under the quintetts between $1.36-1.54 \mathrm{~Hz}$.

${ }^{13} \mathrm{C}\left\{{ }^{1} \mathrm{H}\right\}-\mathrm{NMR}\left(100.6 \mathrm{MHz}, \mathrm{CD}_{2} \mathrm{Cl}_{2}, 295 \mathrm{~K}\right): \delta=25.9\left(\mathrm{CH}_{2}\right), 27.0\left(\mathrm{CH}_{2}\right), 29.5\left(\mathrm{CH}_{2}\right), 29.6\left(C \mathrm{H}_{2}\right), 29.6$ $\left(\mathrm{CH}_{2}\right), 29.7\left(\mathrm{CH}_{2}\right), 29.7\left(\mathrm{CH}_{2}\right), 33.0\left(\mathrm{CH}_{2} \mathrm{CH}_{2} \mathrm{OH}\right), 34.1\left(\mathrm{CH}_{2} \mathrm{CH}_{2} \mathrm{NH}_{2}\right), 42.3\left(\mathrm{CH}_{2} \mathrm{NH}_{2}\right), 62.7$ $\left(\mathrm{CH}_{2} \mathrm{OH}\right)$. 
IR (KBr): $v\left(\mathrm{~cm}^{-1}\right)=3338 \mathrm{~m}, 3086 \mathrm{~m}_{\mathrm{b}}, 2923 \mathrm{~s}, 2849 \mathrm{~s}, 1614 \mathrm{w}, 1472 \mathrm{~m}, 1348 \mathrm{~m}, 1077 \mathrm{~m}, 1031 \mathrm{~m}, 991 \mathrm{~m}$. MS (FAB): $m / z\left(\operatorname{rel}\right.$ int \%) $=188\left([\mathrm{M}+\mathrm{H}]^{+}, 100\right), 69(10), 55(19), 44(9), 41$ (9).

Elemental analysis for $\mathrm{C}_{11} \mathrm{H}_{25} \mathrm{NO}$ calc.: $\mathrm{C} 70.53 \%, \mathrm{H} 13.45 \%$, N 7.48\%; found: $\mathrm{C} 70.69 \%$, $\mathrm{H} 13.43 \%$, N $7.35 \%$.

\section{Synthesis of tert-butyl 11-hydroxyundecylcarbamate}

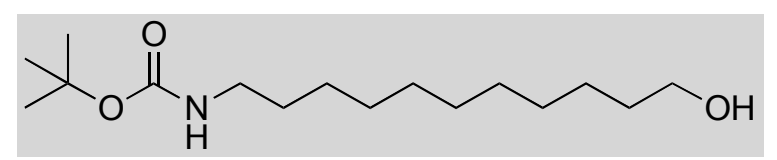

Triethylamine $(9.00 \mathrm{~mL}, 64.7 \mathrm{mmol})$ and di-tert-butyl dicarbonate $(14.1 \mathrm{~g}, 64.6 \mathrm{mmol})$ were added at $0{ }^{\circ} \mathrm{C}$ to a solution of 11 -aminoundecanol $(12.0 \mathrm{~g}, 64.1 \mathrm{mmol})$ in freshly distilled $\mathrm{CH}_{2} \mathrm{Cl}_{2}(200 \mathrm{~mL})$ and the reaction mixture was stirred for 2 hours at $0{ }^{\circ} \mathrm{C}$ and another 8 hours at $23{ }^{\circ} \mathrm{C}$. The resulting solution was washed with a saturated $\mathrm{NaCl}$ solution $(100 \mathrm{~mL})$ and water $(100 \mathrm{~mL})$. The organic phase was dried over $\mathrm{MgSO}_{4}$, filtered and concentrated under reduced pressure. Purification of the crude material by flash chromatography, eluting with $30 \%$ AcOEt in hexanes, gave $89 \%$ yield of tert-butyl 11-hydroxyundecylcarbamate (16.3 g).

m.p.: $34-36{ }^{\circ} \mathrm{C} . \mathrm{R}_{\mathrm{f}}=0.38$ (hexanes/AcOEt $=2: 1$ ).

${ }^{1} \mathrm{H}-\mathrm{NMR}\left(400.1 \mathrm{MHz}, \mathrm{CDCl}_{3}, 295 \mathrm{~K}\right): \delta=1.25\left(\mathrm{~s}_{\mathrm{b}}, 10 \mathrm{H}, \mathrm{CH}_{2}\right), 1.30\left(\mathrm{~m}_{\mathrm{c}}, 4 \mathrm{H}, \mathrm{CH}_{2}\right), 1.42\left(\mathrm{~s}_{\mathrm{b}}, 11 \mathrm{H}\right.$, $\left.\mathrm{C}\left(\mathrm{CH}_{3}\right)_{3}+\mathrm{CH}_{2} \mathrm{CH}_{2} \mathrm{NH}\right), 1.54\left(\mathrm{~m}_{\mathrm{c}}, 3 \mathrm{H}, \mathrm{OH}+\mathrm{CH}_{2} \mathrm{CH}_{2} \mathrm{OH}\right), 3.08\left(\mathrm{~m}_{\mathrm{c}}, J=6.3 \mathrm{~Hz}, 6.6 \mathrm{~Hz}, 2 \mathrm{H}\right.$, $\left.\mathrm{CH}_{2} \mathrm{NH}\right), 3.61\left(\mathrm{~m}_{\mathrm{c}}, J=5.6 \mathrm{~Hz}, 6.3 \mathrm{~Hz}, 2 \mathrm{H}, \mathrm{CH}_{2} \mathrm{OH}\right), 4.54\left(\mathrm{~s}_{\mathrm{b}}, 1 \mathrm{H}, \mathrm{NH}\right)$.

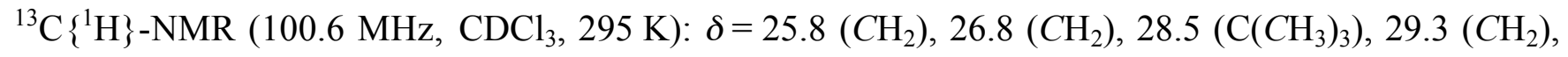
$29.5\left(\mathrm{CH}_{2}\right), 29.5\left(\mathrm{CH}_{2}\right), 29.6\left(\mathrm{CH}_{2}\right), 29.6\left(\mathrm{CH}_{2}\right), 30.1\left(\mathrm{CH}_{2} \mathrm{CH}_{2} \mathrm{NH}\right), 32.8\left(\mathrm{CH}_{2} \mathrm{CH}_{2} \mathrm{OH}\right), 40.7$

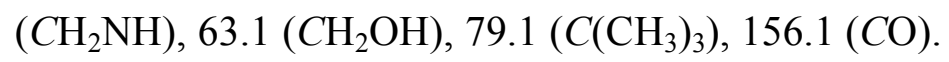

IR (NaCl): $v\left(\mathrm{~cm}^{-1}\right)=3353 \mathrm{~m}_{\mathrm{b}}, 2926 \mathrm{~s}, 2854 \mathrm{~m}, 1693 \mathrm{~s}, 1526 \mathrm{~m}_{\mathrm{b}}, 1366 \mathrm{~m}, 1275 \mathrm{~m}, 1251 \mathrm{~m}, 1173 \mathrm{~s}$.

MS (FAB): $m / z\left(\right.$ rel int \%) = $288\left([\mathrm{M}+\mathrm{H}]^{+}, 3\right), 232$ (7), 188 (17), 74 (12), 69 (11), 57 (100), 41 (26).

Elemental analysis for $\mathrm{C}_{16} \mathrm{H}_{33} \mathrm{NO}_{3}$ calc.: C 66.86\%, H $11.57 \%, \mathrm{~N} 4.87 \%$; found: $\mathrm{C} 66.95 \%, \mathrm{H} 11.49 \%$, N $4.79 \%$.

\section{Synthesis of tert-butyl 11-bromoundecylcarbamate}

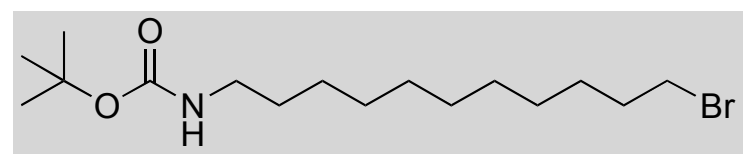

$N$-Bromosuccinimide (10.0 g, $56.2 \mathrm{mmol})$ and triphenylphosphine $(14.8 \mathrm{~g}, 56.4 \mathrm{mmol})$ were added to a solution of tert-butyl 11-hydroxyundecylcarbamate $(16.0 \mathrm{~g}, 55.7 \mathrm{mmol})$ in freshly distilled benzene $(200 \mathrm{~mL})$ and the reaction mixture was stirred for 12 hours at $23{ }^{\circ} \mathrm{C}$. The resulting solution was washed with a saturated $\mathrm{NaCl}$ solution $(200 \mathrm{~mL})$ and water $(200 \mathrm{~mL})$. The organic phase was dried over $\mathrm{MgSO}_{4}$, filtered and concentrated under reduced pressure. Purification by flash chromatography, eluting with $10 \%$ AcOEt in hexanes, gave 74\% yield of tert-butyl 11-bromoundecylcarbamate (14.5 g). 
m.p.: $60-62{ }^{\circ} \mathrm{C} . \mathrm{R}_{\mathrm{f}}=0.37$ (hexanes/AcOEt $\left.=10: 1\right)$.

${ }^{1} \mathrm{H}-\mathrm{NMR}\left(400.1 \mathrm{MHz}, \mathrm{CDCl}_{3}, 295 \mathrm{~K}\right): \delta=1.25\left(\mathrm{~s}_{\mathrm{b}}, 12 \mathrm{H}, \mathrm{CH}_{2}\right), 1.42\left(\mathrm{~s}_{\mathrm{b}}, 13 \mathrm{H}, \mathrm{CH}_{2} \mathrm{CH}_{2} \mathrm{CH}_{2} \mathrm{Br}+\right.$ $\left.\mathrm{C}\left(\mathrm{CH}_{3}\right)_{3}+\mathrm{CH}_{2} \mathrm{CH}_{2} \mathrm{NH}\right), 1.83\left(\mathrm{~m}_{\mathrm{c}}, J=6.9 \mathrm{~Hz}, 7.2 \mathrm{~Hz}, 2 \mathrm{H}, \mathrm{CH}_{2} \mathrm{CH}_{2} \mathrm{Br}\right), 3.08(\mathrm{t}, J=6.6 \mathrm{~Hz}, 2 \mathrm{H}$, $\left.\mathrm{CH}_{2} \mathrm{NH}\right), 3.38$ (t, $\left.J=6.9 \mathrm{~Hz}, 2 \mathrm{H}, \mathrm{CH}_{2} \mathrm{Br}\right), 4.53(\mathrm{~s}, 1 \mathrm{H}, \mathrm{NH})$.

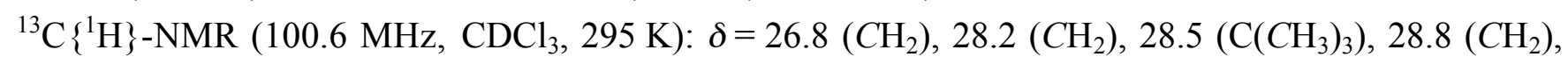
$29.3\left(\mathrm{CH}_{2}\right), 29.4\left(\mathrm{CH}_{2}\right), 29.5\left(\mathrm{CH}_{2}\right), 29.5\left(\mathrm{CH}_{2}\right), 30.1\left(\mathrm{CH}_{2} \mathrm{CH}_{2} \mathrm{NH}\right), 32.9\left(\mathrm{CH}_{2} \mathrm{CH}_{2} \mathrm{Br}\right), 34.1\left(\mathrm{CH}_{2} \mathrm{Br}\right)$, $40.7\left(\mathrm{CH}_{2} \mathrm{NH}\right), 79.0\left(\mathrm{C}\left(\mathrm{CH}_{3}\right)_{3}\right), 156.0(\mathrm{CO})$.

IR $(\mathrm{KBr}): v\left(\mathrm{~cm}^{-1}\right)=3377 \mathrm{~s}, 2918 \mathrm{~s}, 2851 \mathrm{~s}, 1687 \mathrm{~s}_{\mathrm{b}}, 1522 \mathrm{~s}_{\mathrm{b}}, 1469 \mathrm{~m}, 1374 \mathrm{~m}, 1280 \mathrm{~m}, 1237 \mathrm{~m}, 1170 \mathrm{~m}_{\mathrm{b}}$, $1026 \mathrm{w}, 976 \mathrm{w}, 870 \mathrm{~m}, 720 \mathrm{~m}, 643 \mathrm{~m}$.

MS (FAB): $m / z\left(\right.$ rel int \%) = $350\left(\mathrm{M}^{+}, 4,{ }^{79} \mathrm{Br}\right), 294\left(96,{ }^{79} \mathrm{Br}\right), 250\left(13,{ }^{79} \mathrm{Br}\right), 214(8), 137$ (8), 74 (41), 57 (100), $41(20)$.

Elemental analysis for $\mathrm{C}_{16} \mathrm{H}_{32} \mathrm{BrNO}_{2}$ calc.: C 54.85\%, H 9.21\%, N 3.91\%; found: C 55.01\%, H 9.06\%, N $3.89 \%$.

\section{Synthesis of (S)-11-(tert-butoxycarbonyl)undecylethanethioate}

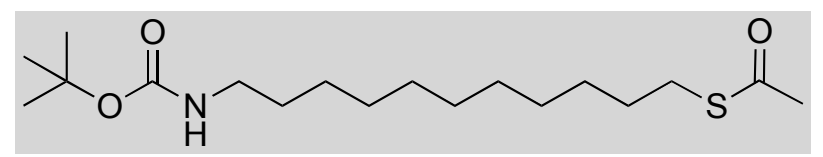

Thioacetic acid $(4.00 \mathrm{~mL}, 56.0 \mathrm{mmol})$, triethylamine $(8.00 \mathrm{~mL}, 57.5 \mathrm{mmol})$ and 4-DMAP (0.50 g, $4.10 \mathrm{mmol})$ were added to a solution of tert-butyl 11-bromoundecylcarbamate (14.5 $\mathrm{g}, 41.4 \mathrm{mmol})$ in freshly distilled $\mathrm{CH}_{2} \mathrm{Cl}_{2}(200 \mathrm{~mL})$ and the reaction mixture was stirred for 24 hours at $23{ }^{\circ} \mathrm{C}$. The solution was washed with a saturated $\mathrm{Na}_{2} \mathrm{CO}_{3}$ solution $(200 \mathrm{~mL})$ and water $(200 \mathrm{~mL})$. The organic phase was dried over $\mathrm{MgSO}_{4}$, filtered and concentrated under reduced pressure. Purification of the crude material by flash chromatography, eluting with 10\% AcOEt in hexanes and subsequent crystallization (n-pentane), gave 79\% yield of $(S)$-11-(tert-butoxycarbonyl)undecyl-ethanethioate (11.3 g).

m.p.: $64-66^{\circ} \mathrm{C} . \mathrm{R}_{\mathrm{f}}=0.25$ (hexanes/AcOEt $=10: 1$ ).

${ }^{1} \mathrm{H}-\mathrm{NMR}\left(400.1 \mathrm{MHz}, \mathrm{CDCl}_{3}, 295 \mathrm{~K}\right): \delta=1.22\left(\mathrm{~s}, 10 \mathrm{H}, \mathrm{CH}_{2}\right), 1.25\left(\mathrm{~m}_{\mathrm{c}}, 2 \mathrm{H}, \mathrm{NCH}_{2} \mathrm{CH}_{2} \mathrm{CH}_{2}\right), 1.30$ $\left(\mathrm{m}_{\mathrm{c}}, 2 \mathrm{H}, \mathrm{CH}_{2} \mathrm{CH}_{2} \mathrm{CH}_{2} \mathrm{~S}\right), 1.41\left(\mathrm{~s}_{\mathrm{b}}, 11 \mathrm{H}, \mathrm{C}\left(\mathrm{CH}_{3}\right)_{3}+\mathrm{NCH}_{2} \mathrm{CH}_{2}\right), 1.52\left(\mathrm{~m}_{\mathrm{c}}, J=6.8 \mathrm{~Hz}, 7.4 \mathrm{~Hz}, 2 \mathrm{H}\right.$, $\left.\mathrm{CH}_{2} \mathrm{CH}_{2} \mathrm{~S}\right), 2.29\left(\mathrm{~s}, 3 \mathrm{H}, \mathrm{SCOCH}_{3}\right), 2.83\left(\mathrm{t}, J=7.4 \mathrm{~Hz}, 2 \mathrm{H}, \mathrm{CH}_{2} \mathrm{~S}\right), 3.07\left(\mathrm{t}, J=6.6 \mathrm{~Hz}, 2 \mathrm{H}, \mathrm{NCH}_{2}\right)$, $4.55\left(\mathrm{~s}_{\mathrm{b}}, 1 \mathrm{H}, \mathrm{N} H\right)$.

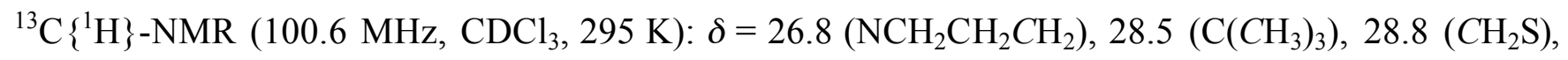
$29.1\left(\mathrm{CH}_{2}\right), 29.2\left(\mathrm{CH}_{2}\right), 29.3\left(\mathrm{CH}_{2} \mathrm{CH}_{2} \mathrm{~S}\right), 29.4\left(\mathrm{CH}_{2}\right), 29.5\left(\mathrm{CH}_{2}\right), 29.5\left(\mathrm{CH}_{2}\right), 29.5\left(\mathrm{CH}_{2}\right), 30.1$ $\left(\mathrm{NCH}_{2} \mathrm{CH}_{2}\right), 30.7\left(\mathrm{SCOCH}_{3}\right), 40.6\left(\mathrm{NCH}_{2}\right), 78.9\left(C\left(\mathrm{CH}_{3}\right)_{3}\right), 156.0(\mathrm{CO}), 196.0(\mathrm{SCO})$.

IR (KBr): $v\left(\mathrm{~cm}^{-1}\right)=3380 \mathrm{~m}, 2918 \mathrm{~m}, 2851 \mathrm{~m}, 1687 \mathrm{~s}_{\mathrm{b}}, 1522 \mathrm{~s}_{\mathrm{b}}, 1469 \mathrm{~m}, 1364 \mathrm{~m}, 1282 \mathrm{~m}, 1238 \mathrm{~m}, 1172 \mathrm{~m}$, $1138 \mathrm{~m}, 1116 \mathrm{~m}, 1000 \mathrm{w}, 959 \mathrm{~m}, 870 \mathrm{w}, 720 \mathrm{w}, 639 \mathrm{~m}$.

MS (FAB): $m / z\left(\right.$ rel int \%) = $346\left([\mathrm{M}+\mathrm{H}]^{+}, 24\right), 290$ (26), 246 (100), 230 (9), 202 (9), 57 (55), 43 (31).

Elemental analysis for $\mathrm{C}_{18} \mathrm{H}_{35} \mathrm{NO}_{3} \mathrm{~S}$ calc.: $\mathrm{C} 62.56 \%, \mathrm{H} 10.20 \%, \mathrm{~N} 4.05 \%$; found: $\mathrm{C} 62.56 \%, \mathrm{H} 10.16 \%$, $\mathrm{N} 4.08 \%$. 


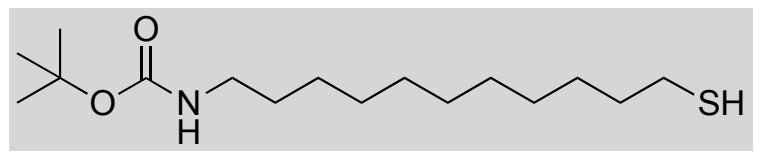

A hydrazine solution ( $1 \mathrm{M}$ in THF, anhydrous, $30.0 \mathrm{~mL}, 30.0 \mathrm{mmol}$ ) was added at $0{ }^{\circ} \mathrm{C}$ to a solution of (S)-11-(tert-butoxycarbonyl)undecylethanethioate (1.00 g, $2.90 \mathrm{mmol})$ in freshly distilled $\mathrm{CH}_{2} \mathrm{Cl}_{2}$ $(20 \mathrm{~mL})$ and the reaction mixture was stirred for 4 hours at $23{ }^{\circ} \mathrm{C}$. The solvent was concentrated under reduced pressure and the residue dissolved in $\mathrm{CH}_{2} \mathrm{Cl}_{2}(50 \mathrm{~mL})$. The resulting solution was washed with a saturated $\mathrm{NH}_{4} \mathrm{Cl}$ solution $(50 \mathrm{~mL})$ and water $(50 \mathrm{~mL})$. The organic phase was dried over $\mathrm{MgSO}_{4}$, filtered and concentrated under reduced pressure. Purification by flash chromatography, eluting with 10\% AcOEt in hexanes, gave $98 \%$ yield of tert-butyl 11-mercaptoundecylcarbamate ( $0.86 \mathrm{~g})$.

m.p.: $52-54{ }^{\circ} \mathrm{C} . \mathrm{R}_{\mathrm{f}}=0.36$ (hexanes/AcOEt $\left.=10: 1\right)$.

${ }^{1} \mathrm{H}-\mathrm{NMR}\left(400.1 \mathrm{MHz}, \mathrm{CDCl}_{3}, 295 \mathrm{~K}\right): \delta=1.25\left(\mathrm{~s}, 12 \mathrm{H}, \mathrm{CH}_{2}\right), 1.32(\mathrm{t}, J=7.6 \mathrm{~Hz}, 1 \mathrm{H}, \mathrm{SH}), 1.36$ $\left(\mathrm{m}_{\mathrm{c}}, 2 \mathrm{H}, \mathrm{CH}_{2} \mathrm{CH}_{2} \mathrm{CH}_{2} \mathrm{SH}\right), 1.43\left(\mathrm{~s}_{\mathrm{b}}, 11 \mathrm{H}, \mathrm{C}\left(\mathrm{CH}_{3}\right)_{3}+\mathrm{NCH}_{2} \mathrm{CH}_{2}\right), 1.59$ (quin, $J=7.2 \mathrm{~Hz}, 2 \mathrm{H}$, $\left.\mathrm{CH}_{2} \mathrm{CH}_{2} \mathrm{~S}\right), 2.51\left(\mathrm{~m}_{\mathrm{c}}, J=7.2 \mathrm{~Hz}, 7.6 \mathrm{~Hz}, 2 \mathrm{H}, \mathrm{CH} \mathrm{H}_{2} \mathrm{SH}\right), 3.09\left(\mathrm{t}, J=6.6 \mathrm{~Hz}, 2 \mathrm{H}, \mathrm{NCH}_{2}\right), 4.50\left(\mathrm{~s}_{\mathrm{b}}, 1 \mathrm{H}\right.$, $\mathrm{N} H)$.

${ }^{13} \mathrm{C}\left\{{ }^{1} \mathrm{H}\right\}$-NMR $\left(100.6 \mathrm{MHz}, \mathrm{CDCl}_{3}, 295 \mathrm{~K}\right): \delta=24.7\left(\mathrm{CH}_{2} \mathrm{SH}\right), 26.9\left(\mathrm{CH}_{2}\right), 28.4\left(\mathrm{CH}_{2} \mathrm{CH}_{2} \mathrm{CH}_{2} \mathrm{SH}\right)$, $28.5\left(\mathrm{C}\left(\mathrm{CH}_{3}\right)_{3}\right), 29.1\left(\mathrm{CH}_{2}\right), 29.3\left(\mathrm{CH}_{2}\right), 29.5\left(\mathrm{CH}_{2}\right), 29.5\left(\mathrm{CH}_{2}\right), 29.6\left(\mathrm{CH}_{2}\right), 30.1\left(\mathrm{NCH}_{2} C \mathrm{H}_{2}\right), 34.1$ $\left(\mathrm{CH}_{2} \mathrm{CH}_{2} \mathrm{SH}\right), 40.7\left(\mathrm{NCH}_{2}\right), 79.0\left(C\left(\mathrm{CH}_{3}\right)_{3}\right), 156.0(\mathrm{CO})$.

IR $(\mathrm{KBr}): v\left(\mathrm{~cm}^{-1}\right)=3373 \mathrm{~s}, 2921 \mathrm{~s}, 2848 \mathrm{~s}, 1682 \mathrm{~s}_{\mathrm{b}}, 1513 \mathrm{~s}_{\mathrm{b}}, 1464 \mathrm{~m}, 1367 \mathrm{~m}, 1284 \mathrm{~m}, 1241 \mathrm{~m}, 1166 \mathrm{~s}_{\mathrm{b}}$, $1044 \mathrm{w}, 1001 \mathrm{~m}, 977 \mathrm{~m}, 864 \mathrm{~m}, 783 \mathrm{w}, 723 \mathrm{~m}, 580 \mathrm{~m}_{\mathrm{b}}$.

MS (FAB): $m / z\left(\right.$ rel int \%) = $304\left([\mathrm{M}+\mathrm{H}]^{+}, 10\right), 248$ (41), 204 (100), 74 (13), 57 (77), 41 (14).

Elemental analysis for $\mathrm{C}_{16} \mathrm{H}_{33} \mathrm{NO}_{2} \mathrm{~S}$ calc.: $\mathrm{C} 63.32 \%, \mathrm{H} 10.96 \%, \mathrm{~N} 4.62 \%$, O 10.54\%; found: $\mathrm{C} 63.41 \%$, H $10.90 \%$, N $4.62 \%$, O $10.46 \%$.

\section{Synthesis of 11-aminoundecane-1-thiol hydrochloride}

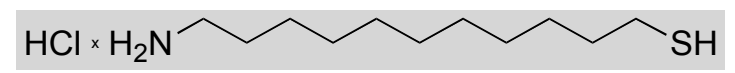

Tert-butyl 11-mercaptoundecylcarbamate $(1.00 \mathrm{~g}, 3.30 \mathrm{mmol})$ was dissolved in a $\mathrm{HCl}$ solution in dioxane $(4 \mathrm{M}, 20.0 \mathrm{~mL}, 80 \mathrm{mmol})$ and the reaction mixture was stirred for 3 hours at $23{ }^{\circ} \mathrm{C}$. The solvent was concentrated under reduced pressure and the solid washed with diethyl ether $(50 \mathrm{~mL})$ and $n$-pentane $(50 \mathrm{~mL})$ to provide the desired 11-aminoundecane-1-thiol hydrochloride $(0.78 \mathrm{~g})$ in $99 \%$ yield. m.p.: $162-164{ }^{\circ} \mathrm{C}$.

${ }^{1} \mathrm{H}-\mathrm{NMR}\left(400.1 \mathrm{MHz},\left(\mathrm{CD}_{3}\right)_{2} \mathrm{SO}, 295 \mathrm{~K}\right): \delta=1.25\left(\mathrm{~s}, 12 \mathrm{H}, \mathrm{CH}_{2}\right), 1.32\left(\mathrm{~m}_{\mathrm{c}}, 2 \mathrm{H}, \mathrm{NCH}_{2} \mathrm{CH}_{2} \mathrm{CH}_{2}\right)$, 1.51 (quin, $J=6.8 \mathrm{~Hz}, 2 \mathrm{H}, \mathrm{CH}_{2} \mathrm{CH}_{2} \mathrm{SH}$ ), 1.53 (quin, $J=7.4 \mathrm{~Hz}, 2 \mathrm{H}, \mathrm{NCH}_{2} \mathrm{CH}_{2}$ ), $2.23(\mathrm{t}, J=7.6 \mathrm{~Hz}$, $1 \mathrm{H}, \mathrm{SH}), 2.46\left(\mathrm{~m}_{\mathrm{c}}, J=6.8 \mathrm{~Hz}, 7.6 \mathrm{~Hz}, 2 \mathrm{H}, \mathrm{CH}_{2} \mathrm{SH}\right), 2.75\left(\mathrm{t}_{\mathrm{b}}, J=7.4 \mathrm{~Hz}, 2 \mathrm{H}, \mathrm{NCH}_{2}\right), 7.78\left(\mathrm{~s}_{\mathrm{b}}, 3 \mathrm{H}\right.$, $\left.\mathrm{NH} H_{3} \mathrm{Cl}\right)$. 
${ }^{13} \mathrm{C}\left\{{ }^{1} \mathrm{H}\right\}$-NMR $\left(100.6 \mathrm{MHz},\left(\mathrm{CD}_{3}\right)_{2} \mathrm{SO}, 295 \mathrm{~K}\right): \delta=23.7\left(\mathrm{CH}_{2} \mathrm{SH}\right), 25.8\left(\mathrm{CH}_{2}\right), 26.9\left(\mathrm{NCH}_{2} \mathrm{CH}_{2}\right), 27.7$ $\left(\mathrm{NCH}_{2} \mathrm{CH}_{2} \mathrm{CH}_{2}\right), 28.5\left(\mathrm{CH}_{2}\right), 28.5\left(\mathrm{CH}_{2}\right), 28.8\left(\mathrm{CH}_{2}\right), 28.9\left(\mathrm{CH}_{2}\right), 28.9\left(\mathrm{CH}_{2}\right), 33.4\left(\mathrm{CH}_{2} \mathrm{CH}_{2} \mathrm{SH}\right), 38.7$ $\left(\mathrm{NCH}_{2}\right)$.

IR (KBr): $v\left(\mathrm{~cm}^{-1}\right)=2918 \mathrm{~s}_{\mathrm{b}}, 2657 \mathrm{~m}, 2547 \mathrm{~m}, 2021 \mathrm{w}_{\mathrm{b}}, 1625 \mathrm{~m}, 1511 \mathrm{~m}, 1473 \mathrm{~m}, 1398 \mathrm{w}, 1308 \mathrm{w}, 1196 \mathrm{w}$. MS (FAB): $\left.m / z(\text { rel int \%) }=204 \text { ([M - Chlorid }]^{+}, 100\right), 55$ (6).

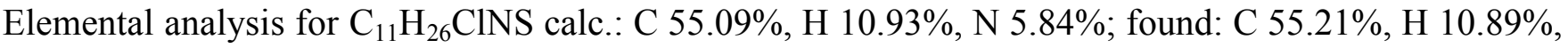
N 5.73\%.

\section{Analyses of thiolate-protected gold colloids}

\begin{tabular}{|c|c|c|}
\hline Colloid & d (TEM) $[\mathrm{nm}]$ & Elemental analysis \\
\hline $\mathbf{6}$ & $2.32 \pm 0.46 \mathrm{~nm}$ & $\mathrm{C} 16.52 \%, \mathrm{~S} 2.29 \%, \mathrm{Au} 79.5 \%$ \\
\hline $\mathbf{7}$ & $3.48 \pm 0.61 \mathrm{~nm}$ & $\mathrm{C} 7.15 \%, \mathrm{~S} 2.38 \%, \mathrm{Au} 90.1 \%$ \\
\hline $\mathbf{8}$ & $3.44 \pm 0.59 \mathrm{~nm}$ & $\mathrm{C} 11.39 \%, \mathrm{~S} 2.22 \%, \mathrm{Au} 76.8 \%$ \\
\hline $\mathbf{9}$ & $2.60 \pm 0.43 \mathrm{~nm}$ & $\mathrm{C} 7.39 \%, \mathrm{~N} 0.71, \mathrm{~S} 1.40 \%, \mathrm{Au} 81.5 \%$ \\
\hline $\mathbf{1 0}$ & $3.39 \pm 0.73 \mathrm{~nm}$ & $\mathrm{C} 8.26 \%, \mathrm{O} 0.83, \mathrm{~S} 1.79 \%, \mathrm{Au} 79.3 \%$ \\
\hline
\end{tabular}

\section{TEM-image of colloid 7}

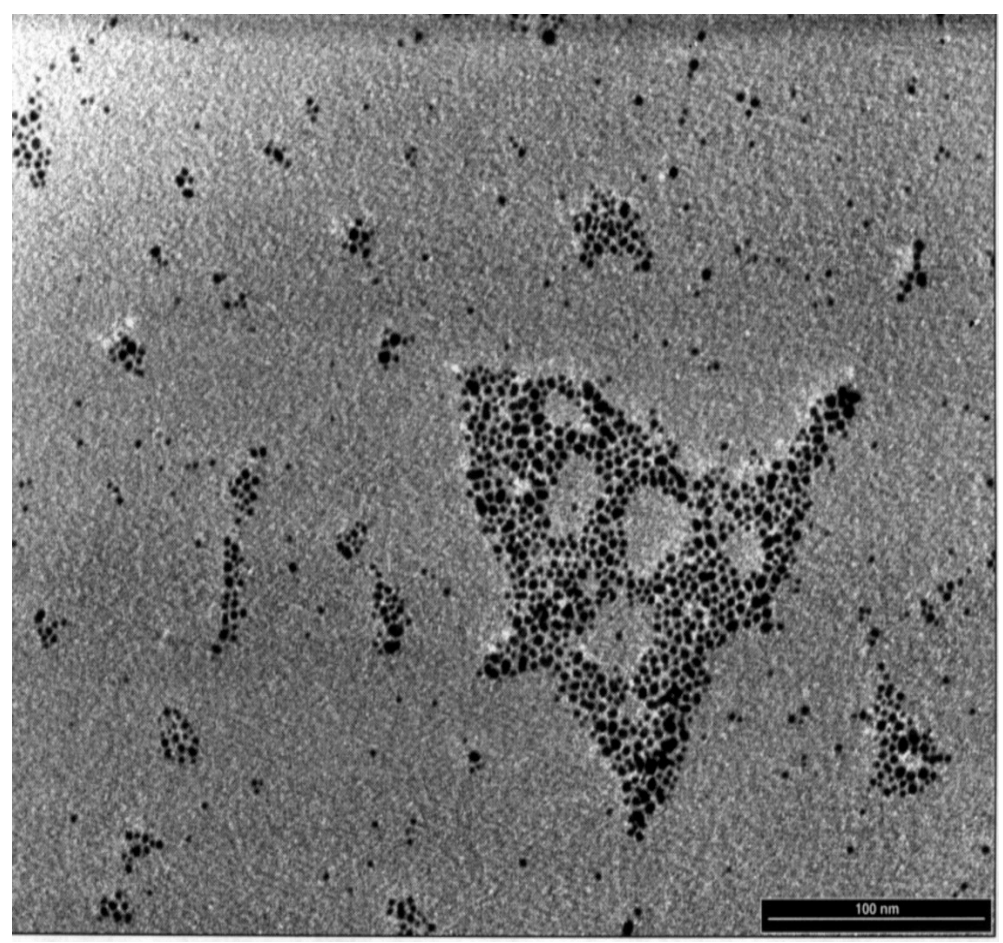




\section{Complete Reference 13:}

[13] M. J. Hostetler, J. E. Wingate, C.-J. Zhong, J. E. Harris, R. W. Vachet, M. R. Clark, J. D. Londono, S. J. Green, J. J. Stokes, G. D. Wignall, G. L. Glish, M. D. Porter, N. D. Evans, R. W. Murray, Langmuir 1998, 14, 17. 